\title{
The Psychosocial Reactions to COVID-19 Pandemic among Individuals in the Arab World
}

\author{
Souad Mousa Ahmed Bakhet \\ Assoc. Prof. in Health and Clinical Psychology, \\ Ahfad University for Women, Sudan \\ Rasis Abdullah Alanazi \\ School of Psychology, Princess Nourah Bint Abdulrahman \\ University, Riyadh, Saudi Arabia \\ Rehab Abdullahmeed Nori \\ School of Psychology, Princess Nourah Bint Abdulrahman \\ University, Riyadh, Saudi Arabia
}

\section{Abstract}

This current study aimed at investigating the psycho social reaction to covid19 among individual in the Arab world. In addition, to discover the relationship between these reactions with various variables like age, gender, educational level, occupation and place of origin and place of residence. An open-end questions set by the researchers distributed via the internet. Five hundred and twenty eight (528) respondents answer the questions, 398 females and 130 males. The result showed that the majority of the respondent never or rarely feel negative psychosocial reactions in the entire question except feeling anxious and Suspicions of any person sneeze and this sound logic because the majority of the respondents have no experience of covid19. Thus, they are not under the pressure of suffering from this dangerous and threating disease. The majority of participants from Saudi and Sudan, and other United Emirate country, simply because the researchers from these two countries. Covid19 major concern are physical aspect of the disease, no one can doubt the importance of the psychosocial issues, which are the concern of this study. Other aspect the religious background of the Arabic country especially Sudan and Saudi are conservative and culture restricted communities, thus their psychosocial reaction colored with these background. This study cannot be generalized to the completely Arabic world but it can give an indicator on how individual in the Arabic world think, feel, and react. The study end with conclusion and some recommendations.

Keywords: Covid-19 pandemic, psychosocial reactions, and Arab world 


\section{Introduction}

Coronavirus 2019 (covid-19) is a highly infectious disease caused by a new coronavirus. The disease discovered in china in December 2019 and then spread around the world, causing an unprecedented public health crisis. Aaron Kandola, 2020).With the global breakout of the disease, many concerns raised to identify its threats on people's health. It is consider as a threat due to fast spread and transmitted by human-to-human interaction. COVID-19 is still under research but it has been related to human severe acute respiratory syndrome (SARS) that occurred in 2003 and Middle East respiratory syndrome (MERS) (Zhu et al., 2020).Several physical symptoms have been associated to COVID-19 which includes fever, cough, fatigue, headache, hemoptysis and diarrhea (Torales eta., 2020). It is important to note that due to the fast nature of the outbreak, many people may suffer from different psychological symptoms. For example, people during the epidemic may report having anxiety, depression, panic attacks, and insomnia. On a social level, individuals may feel isolated due to lockdown in many countries and quarantine. Pertaining to economy, many trades were paused and more restrictions were applied which in turn affected the economy worldwide. The amount of impact of the epidemic have led many to practice spiritual and religious acts to overcome the outbreak spiritually. It is vital to understand the public psychosocial states and reactions to the epidemic to help in providing further evidence and research to establish better global health measures that addresses different stressors to encompass the disease.

\section{Objectives:}

The goal of this study is to discover the Psychosocial reactions to epidemic COVID-19 in relation to individuals' Age, gender, socioeconomic status, occupation and place of origin. The study will help to improve current and future interventions as a response to future pandemics.

\section{Review of the Literature}

Corona virus 2019 (Covid19) is an infectious disease that affect the respiratory system of the human being. It started in China and then spread all over the world, in Europe, Asia, America, Middle East and Africa. Several ongoing researches had been running in different part of the world, the main concern about the symptoms of the disorder, the ways of transmission, the incubation period, the prevention and treatment. Even those studies under research and still are not defiantly accurate. However, this disorders and due to it prevention methods, which mainly concentrate on social isolation and detachment, which against the nature of the human being, will affect the various component of the individual. Namely, the psychological, the social, spiritual, economic, political, and culture aspect of an individual. In the Arabic world although several common issues tight them, but still there might be different reactions to covid19 the researchers expected in particular the psychosocial reactions. The psychological reactions such as fear and anxiety, tension and 
uncertainty, trauma, depression, grief, confusion, social phobia and obsession and compulsion among others. The social reaction like social isolation, stigma, violence, fear of unknown, loneliness, panic, lack of control, lack of information, and wrong information, and malicious websites (Hackers). The economic reactions due to social isolation program may lead to loss of jobs. Schools and universities closed which will delay the graduate dates of students and then affect the workforce. Countries with low economic status, covd19 aggravates their poor conditions. New industries will appear in the domain of health, clean and hygiene, the innovate ideas of making masks and create activities when family members at home. Political reactions, the conflicting interest and hidden agenda for power balance and who want to lead the world. There is on-going research that examines the physical aspect of COVID-19 and symptoms of the virus. However, there is a need to identify the psychological and social aspects of the epidemic. In a study conducted by Hawryluck et al in (2004) in Canada by using a cross-sectional, design on 129 Toronto residents to assess post-traumatic stress and depression. Results indicated that the surveyed sample have experienced high prevalence of psychological distress and Individual have suffered posttraumatic stress disorder by $28.9 \%$ of participants. In another study Conducted by Hong et al., (2009) to measure the impact of severe acute respiratory syndrome (SARS) in 2002 on patients. They recruited 70 subjects that had suffered from SARA at different stages after being treated. They examined the subjects by conducting a clinical assessment of posttraumatic disorder in five different stages. Several surveys were use in the study that included Impact of Event Scale (IES), Zung Self-Rating Anxiety Scale (SAS), Zung Self-Rating Depression Scale (SDS), Symptom Checklist 90 (SCL-90), Short Form-36 (SF-36 Health Survey) and Social Disability Screening Schedule (SDSS). Results indicated that out of the 68 subjects 30 have developed PTSD.During the SARS outbreak, several issues highlighted directly or indirectly emerged from the disease. Psychological issues were one issue that researchers have thoroughly examined.Nevertheless, other social issues reported as part of the direct impact of quarantine such as stigma. It found in the previous literature that stigma was a major theme during the outbreak. Many health workers and patients have been more likely to report stigmatization from people around them. Studies have showed that participants from health workers and patients were more likely to experience avoidance, lack of social invitations, and treating them differently. As a result, several health-care workers have suffered from household tension due to their family consideration of their jobs being high at risk. (Cave et al., 2005, Desclaux et al., 2017; DiGiovanni et al., 2004; Hawryluck et al., 2004). Many studies carried in the area of mental health with covid19, a study conducted by Nilima, N., Kaushik, S., Tiwary, B., \& Pandey, P. K. (2021) on the psycho-social factors associated with COVID-19 and the nationwide lockdown in India. It was a cross-sectional online survey conducted among the people of all states in India. A spatial analysis was performed and Moran's I statistic was applied to investigate the overall clustering of locations. Fisher's exact test used to investigate associations. One thousands three hundred and sixteen (1316) responses were received. The results showed those worried for their family's 
health were likely to follow the lockdown measures ( $\mathrm{p}<0.001)$.Significant association was observed $(\mathrm{p}<0.001)$ between following the lockdown measures and being satisfied with the government strategy to combat the COVID-19 pandemic. A significant relation was observed between the gender $(p=0.001)$, job profile $(\mathrm{p}<0.001)$ and physical activity $(\mathrm{p}<0.001)$ were observed to be associated with the psychosocial impact. The study Conclude that, Government and public health officials should consider the sentiments of the community while planning strategies relating to the pandemic. The findings of this study will assist the policymakers in emphasizing the psychological well-being of individuals, along with physical health. They find that, the increase in death and spread-related coronavirus (COVID-19) has shifted the world focus to the containment of the disease by emphasizing measures to prevent spread in the general population. Such a complex, threatening, and unprecedented situation has left the psychosocial wellbeing needs of public unaddressed. This paper aims to review the current COVID-19 scenario and its effects on the psychosocial wellbeing of people; and an attempt to shed some light on the aforementioned questions. Furthermore, the review will propose some recommendations for overcoming the mental illness issues, during and after the COVID-19 outbreak. We extracted information from reliable published international and national literature and reviewed anecdotes from media content from January to June 2020. The mental health implications of this outbreak will be long lasting; however, by prioritizing, investing diligently, and taking a collective approach, this challenge dealt with in a promising manner. Artificial intelligence in prediction of mental health disorders induced by the COVID-19 pandemic among health care workers. immediate aftermath present a serious threat to the mental health of care workers (HCWs), who may develop elevated rates of anxiety, depression, posttraumatic stress disorder, or even suicidal behaviors. The proposed methodology for prediction of mental health disorders among HCWs during the ongoing pandemic based on AI-aided data analysis is particularly important since they are a high-risk group for contracting the COVID-19 disease (68) and developing later stress-related symptoms. However, the methodology proposed in this article might be applied generally for all those who were exposed to higher levels of such risks during the COVID-19 pandemic. Public psychology is often very fragile during outbreaks of infectious diseases. Amid a lack of psychological endurance in the public, inappropriate or excessive release of panic-triggering information by television, radio, newspaper, magazine, Internet, and other media sources can have a negative impact on the population. From the perspective of communication psychology, during such times, people tend to carry an affinity for negative, threatening, or informal information and for ignoring or doubting positive and formal information. Furthermore, during disease outbreaks, rumors and speculation abound; authorities in endemic areas take emergency measures, such as sealing cities and roads; streets become quiet; the economy can struggle; the stock market is instable. There is a rush for supplies and household items; pedestrians remain heavily protected and armed; and advertisements and posters on the disease ubiquitously posted, all of which can 
make people feel nervous and scared. The COVID-19 virus is a serious threat to the health and life of patients themselves, as well as the surrounding population given its short incubation period, rapid onset, rapid change, strong infectivity. Therefore, the disease itself is a stressor, causing excessive and continuous stress and possibly leading to mental disorders, primarily manifested as anxiety and depression.

Methods:

A descriptive method of research applied in this study,

Population and sample:

All the individual live in the Arabic world will targeted to the study, those who will willingly filling and answering the questions will be the sample of the study.

Tool of data collection: an open end questions had designed by the researchers, the questions include the demographic variables (gender, age, educational level, occupation, marital status, socioeconomic status, and place of residence and nationality), the other part of the questions about the psychological and social reactions to covid19 as expected from the respondents.

Reliability of the psychosocial reactions to covid19 questions

\begin{tabular}{|l|l|}
\hline Cronbach's Alpha & No of Items \\
\hline .751 & 21 \\
\hline
\end{tabular}

The above table shows the reliability of the questions that designed for the psychosocial reactions to covid 19 which the alpha Cronbach .751 . The placed online in the internet for a month and a half period, 528 respondents will received 398 females and 130 are males.

Data analysis: correlation coefficient and analysis of variance used for data analysis.

\section{Results}

Table NO 1. Gender of the respondents:

\begin{tabular}{|l|l|l|}
\hline & Frequency & Percent \\
\hline Female & 398 & 75.4 \\
Male & 130 & 24.6 \\
Total & 528 & 100.0 \\
\hline
\end{tabular}

Table one above showed the gender of the respondents, the majority were females 398 while only 130 males.

Table NO 2. Age of the respondents: 


\begin{tabular}{|l|l|l|}
\hline & Frequency & Percent \\
\hline 0-20 year & 39 & 7.4 \\
21-30 year & 120 & 22.7 \\
31-40 year & 121 & 22.9 \\
41-50 year & 128 & 24.2 \\
51-60 year & 92 & 17.4 \\
61-70 year & 23 & 4.4 \\
71 more & 5 & .9 \\
Total & 528 & 100.0 \\
& & \\
\hline
\end{tabular}

Table two showed the age of the respondents, $24.2 \%$ from age droup 41-50, 22.9\% from $31-40,22.7 \%$ from $21-30,17.4 \%$ from $51-60$ age group the rest few are under 21 and over 71 year old.

Table NO 3. Place of origin of the respondents:

\begin{tabular}{|l|l|l|}
\hline & Frequency & Percent \\
\hline Rural & 12 & 2.3 \\
Urban & 516 & 97.7 \\
Total & 528 & 100.0 \\
\hline
\end{tabular}

Table 3 above showed the majority of the respondent their place of origin is urban areas they constitute $97.7 \%$ while only $2.3 \%$ are from rural areas.

Table NO 4. The occupation of the respondents:

\begin{tabular}{|l|l|l|}
\hline & Frequency & Percent \\
\hline Employee & 84 & 15.9 \\
Housewife & 126 & 23.9 \\
Private Sector & 71 & 13.4 \\
Public Sector & 30 & 5.7 \\
Student & 110 & 20.8 \\
Teacher & 67 & 12.7 \\
Without & 37 & 7.0 \\
Work & 3 & .6 \\
Worker & 528 & 100.0 \\
Total & & \\
\hline
\end{tabular}

Table 4 above showed that $23.9 \%$ of the respondents are housewives, $20.8 \%$ are students, $15.9 \%$ are employee, $13.4 \%$ work in private sector, $12.7 \%$ are teachers, $7 \%$ without work, $5.7 \%$ work in the public sector and .6\% are workers. 
Table No 5. Place of residence:

\begin{tabular}{|l|l|l|}
\hline & Frequency & Percent \\
\hline Other & 144 & 27.3 \\
Saudi Arabia & 196 & 37.1 \\
Sudan & 188 & 35.6 \\
Total & 528 & 100.0 \\
\hline
\end{tabular}

Table five above showed the place of residence for the respondents, $37.1 \%$ from Saudi Aribia, $35.6 \%$ from Sudan and $27.3 \%$ are from other countries (united emirate and others).

Table NO 6,. Age * score

Descriptive

\begin{tabular}{|c|c|c|c|c|c|c|c|c|}
\hline \multicolumn{9}{|l|}{ Score } \\
\hline & \multirow[b]{2}{*}{$\mathrm{N}$} & \multirow[b]{2}{*}{ Mean } & \multirow[b]{2}{*}{$\begin{array}{l}\text { Std. } \\
\text { Deviation }\end{array}$} & \multirow[b]{2}{*}{$\begin{array}{l}\text { Std. } \\
\text { Error }\end{array}$} & \multicolumn{2}{|c|}{$\begin{array}{l}95 \% \text { Confidence } \\
\text { Interval for Mean }\end{array}$} & \multirow[b]{2}{*}{$\begin{array}{l}\text { Minim } \\
\text { um }\end{array}$} & \multirow[b]{2}{*}{$\begin{array}{l}\text { Maxi } \\
\text { mum }\end{array}$} \\
\hline & & & & & $\begin{array}{l}\text { Lower } \\
\text { Bound }\end{array}$ & $\begin{array}{l}\text { Upper } \\
\text { Bound }\end{array}$ & & \\
\hline $\begin{array}{l}20 \text { or } \\
\text { Less }\end{array}$ & 39 & 22.51 & 11.630 & 1.862 & 18.74 & 26.28 & 1 & 45 \\
\hline $\begin{array}{l}21-30 \\
\text { years }\end{array}$ & 119 & 20.24 & 11.292 & 1.035 & 18.19 & 22.29 & 0 & 47 \\
\hline $\begin{array}{l}31-40 \\
\text { years }\end{array}$ & 123 & 16.19 & 11.270 & 1.016 & 14.18 & 18.20 & 0 & 51 \\
\hline $\begin{array}{l}41-50 \\
\text { years }\end{array}$ & 128 & 18.75 & 10.246 & .906 & 16.96 & 20.54 & 2 & 44 \\
\hline $\begin{array}{l}51-60 \\
\text { years }\end{array}$ & 93 & 18.26 & 11.399 & 1.182 & 15.91 & 20.61 & 0 & 53 \\
\hline $\begin{array}{l}61-70 \\
\text { years }\end{array}$ & 23 & 14.83 & 11.555 & 2.409 & 9.83 & 19.82 & 0 & 40 \\
\hline $\begin{array}{l}71 \text { and } \\
\text { above }\end{array}$ & 5 & 7.40 & 6.189 & 2.768 & -.28 & 15.08 & 2 & 18 \\
\hline Total & 530 & 18.40 & 11.224 & .488 & 17.45 & 19.36 & 0 & 53 \\
\hline
\end{tabular}


ANOVA

\begin{tabular}{|l|l|l|l|l|l|}
\cline { 2 - 6 } \multicolumn{1}{c|}{ Score } & & & & & \\
\hline & Sum of Squares & Df & Mean Square & F & Sig. \\
\hline $\begin{array}{l}\text { Between } \\
\text { Groups } \\
\text { Within } \\
\text { Groups } \\
\text { Total }\end{array}$ & 2582.906 & 6 & 430.484 & 3.515 & .002 \\
\hline
\end{tabular}

Table 6 above showed that there is significant differences in the psychosocial reaction to covid19 in age group in favour of 51-60 years old df 6 and p .002 .

Table NO 7, Place of origin

Descriptive

\begin{tabular}{|c|c|c|c|c|c|c|c|c|}
\hline \multicolumn{9}{|l|}{$\begin{array}{l}\text { Sco } \\
\text { re }\end{array}$} \\
\hline & \multirow[b]{2}{*}{$\mathrm{N}$} & \multirow[b]{2}{*}{ Mean } & \multirow[b]{2}{*}{$\begin{array}{l}\text { Std. } \\
\text { Deviatio } \\
\mathrm{n}\end{array}$} & \multirow[b]{2}{*}{ Std. Error } & \multicolumn{2}{|c|}{$\begin{array}{l}\text { 95\% Confidence } \\
\text { Interval for Mean }\end{array}$} & \multirow[b]{2}{*}{ Minimum } & \multirow[b]{2}{*}{$\begin{array}{l}\text { Maximı } \\
\mathrm{m}\end{array}$} \\
\hline & & & & & Lower Bound & $\begin{array}{l}\text { Upp } \\
\text { er } \\
\text { Bou } \\
\text { nd }\end{array}$ & & \\
\hline $\begin{array}{l}\text { Urb } \\
\text { an }\end{array}$ & $\begin{array}{l}51 \\
8\end{array}$ & 18.23 & 11.205 & .492 & 17.26 & $\begin{array}{l}19.2 \\
0\end{array}$ & 0 & 53 \\
\hline $\begin{array}{l}\text { Rur } \\
\text { al }\end{array}$ & 12 & 25.92 & 9.690 & 2.797 & 19.76 & $\begin{array}{l}32.0 \\
7\end{array}$ & 12 & 41 \\
\hline $\begin{array}{l}\text { Tot } \\
\text { al }\end{array}$ & $\begin{array}{l}53 \\
0\end{array}$ & 18.40 & 11.224 & .488 & 17.45 & $\begin{array}{l}19.3 \\
6\end{array}$ & 0 & 53 \\
\hline
\end{tabular}

ANOVA

\begin{tabular}{|c|c|c|c|c|c|}
\hline \multicolumn{6}{|l|}{ Score } \\
\hline & Sum of Squares & Df & Mean Square & $\mathrm{F}$ & Sig. \\
\hline $\begin{array}{l}\text { Between } \\
\text { Groups }\end{array}$ & 693.014 & 1 & & 5.549 & .019 \\
\hline $\begin{array}{l}\text { Within } \\
\text { Groups }\end{array}$ & 65946.579 & 528 & & & \\
\hline Total & 66639.592 & 529 & & & \\
\hline
\end{tabular}


Table 7 above showed that there is a significant difference in psychosocial reaction to covid19 in the place of origin of the respondents in favour of urban origin, df 1 p.019.

Table NO 8. Occupation

Descriptive

\begin{tabular}{|c|c|c|c|c|c|c|c|c|}
\hline \multicolumn{9}{|l|}{ Score } \\
\hline & \multirow[b]{2}{*}{$\mathrm{N}$} & \multirow[b]{2}{*}{ Mean } & \multirow[b]{2}{*}{$\begin{array}{l}\text { Std. } \\
\text { Deviation }\end{array}$} & \multirow[b]{2}{*}{$\begin{array}{l}\text { Std. } \\
\text { Error }\end{array}$} & \multicolumn{2}{|c|}{$\begin{array}{l}95 \% \text { Confidence } \\
\text { Interval for Mean }\end{array}$} & \multirow[b]{2}{*}{$\begin{array}{l}\text { Minim } \\
\text { um }\end{array}$} & \multirow[b]{2}{*}{$\begin{array}{l}\text { Maxim } \\
\text { um }\end{array}$} \\
\hline & & & & & $\begin{array}{l}\text { Lower } \\
\text { Bound }\end{array}$ & $\begin{array}{l}\text { Upper } \\
\text { Bound }\end{array}$ & & \\
\hline Teacher & 67 & 18.31 & 11.803 & 1.442 & 15.43 & 21.19 & 2 & 50 \\
\hline Student & 110 & 21.57 & 11.920 & 1.137 & 19.32 & 23.83 & 0 & 47 \\
\hline $\begin{array}{l}\text { house } \\
\text { wife }\end{array}$ & 125 & 17.93 & 10.601 & .948 & 16.05 & 19.80 & 0 & 51 \\
\hline $\begin{array}{l}\text { Private } \\
\text { Sector }\end{array}$ & 71 & 15.27 & 9.980 & 1.184 & 12.91 & 17.63 & 0 & 53 \\
\hline $\begin{array}{l}\text { Public } \\
\text { sector }\end{array}$ & 30 & 19.20 & 10.545 & 1.925 & 15.26 & 23.14 & 4 & 39 \\
\hline $\begin{array}{l}\text { Unemplo } \\
\text { yed }\end{array}$ & 37 & 19.08 & 12.670 & 2.083 & 14.86 & 23.31 & 3 & 46 \\
\hline Employer & 84 & 17.62 & 10.205 & 1.113 & 15.40 & 19.83 & 0 & 43 \\
\hline Labour & 3 & 5.00 & 7.000 & 4.041 & -12.39 & 22.39 & 0 & 13 \\
\hline Other & 3 & 17.33 & 20.526 & $\begin{array}{l}11.85 \\
1\end{array}$ & -33.66 & 68.32 & 0 & 40 \\
\hline Total & 530 & 18.40 & 11.224 & .488 & 17.45 & 19.36 & 0 & 53 \\
\hline
\end{tabular}

ANOVA

\begin{tabular}{|l|l|l|l|l|l|}
\cline { 2 - 6 } \multicolumn{1}{l|}{ Score } & & & & & \\
\hline & Sum of Squares & $\mathrm{df}$ & $\begin{array}{l}\text { Mean } \\
\text { Square }\end{array}$ & F & Sig. \\
\hline $\begin{array}{l}\text { Between } \\
\text { Groups } \\
\begin{array}{l}\text { Within } \\
\text { Groups } \\
\text { Total }\end{array}\end{array}$ & 2461.956 & 8 & 307.744 & 2.498 & .011 \\
\hline
\end{tabular}

Table 8 above showed that there is a significance differences in psychosocial reaction to covid19 in terms of occupation among the respondents in favour to private sector, df 8 p.011. 
Table NO 9, Place of residence:

Descriptive

\begin{tabular}{|c|c|c|c|c|c|c|c|c|}
\hline \multicolumn{9}{|l|}{ Score } \\
\hline & \multirow[b]{2}{*}{$\mathrm{N}$} & \multirow[b]{2}{*}{ Mean } & \multirow[b]{2}{*}{$\begin{array}{l}\text { Std. } \\
\text { Deviation }\end{array}$} & \multirow[b]{2}{*}{$\begin{array}{l}\text { Std. } \\
\text { Error }\end{array}$} & \multicolumn{2}{|c|}{$\begin{array}{l}95 \% \text { Confidence } \\
\text { Interval for Mean }\end{array}$} & \multirow[b]{2}{*}{$\begin{array}{l}\text { Minim } \\
\text { um }\end{array}$} & \multirow[b]{2}{*}{$\begin{array}{l}\text { Maxim } \\
\text { um }\end{array}$} \\
\hline & & & & & $\begin{array}{l}\text { Lower } \\
\text { Bound }\end{array}$ & $\begin{array}{l}\text { Upper } \\
\text { Bound }\end{array}$ & & \\
\hline Sudan & 189 & 18.57 & 10.722 & .780 & 17.03 & 20.10 & 0 & 53 \\
\hline $\begin{array}{l}\text { Saudi } \\
\text { Arabia }\end{array}$ & 197 & 18.86 & 11.372 & .810 & 17.27 & 20.46 & 0 & 47 \\
\hline Other & 144 & 17.56 & 11.687 & .974 & 15.64 & 19.49 & 0 & 51 \\
\hline Total & 530 & 18.40 & 11.224 & .488 & 17.45 & 19.36 & 0 & 53 \\
\hline
\end{tabular}

\section{ANOVA}

\begin{tabular}{|l|l|l|l|l|l|}
\cline { 2 - 6 } \multicolumn{1}{l|}{ Score } & & & & & \\
\hline & Sum of Squares & Df & Mean Square & F & Sig. \\
\hline Between & 148.432 & 2 & 74.216 & .588 & .556 \\
Groups & 66491.160 & 527 & 126.169 & & \\
Within Groups & 66639.592 & 529 & & & \\
Total & 6 & & & \\
\hline
\end{tabular}

Table 9 above showed there is no significance difference in psychosocial reaction to covide19 in terms of place of residence among the respondents.

Table NO 10, Relation to covid19

\begin{tabular}{|l|l|l|}
\hline & Frequency & Percent \\
\hline $\begin{array}{l}\text { Have no } \\
\text { disease } \\
\text { Co patient } \\
\text { to covid19 } \\
\text { Live with } \\
\text { covid19 } \\
\text { patient } \\
\text { Infected } \\
\text { with } \\
\text { covid19 }\end{array}$ & 515 & 97.5 \\
Total & 1 & .9 \\
\hline
\end{tabular}

Table 10 above showed the almost the majority have no covid19 infection $97.5 \%$, 
$1.3 \%$ are co patient, $.9 \%$ live with covid 19 patient and only .2\% are infected.

Table (12)

The psychosocial reactions:

\begin{tabular}{|c|c|c|c|c|c|c|c|c|c|c|c|c|c|c|}
\hline $\begin{array}{l}\text { Psychos } \\
\text { ocial } \\
\text { reaction } \\
\text { s }\end{array}$ & $\begin{array}{l}\text { alway } \\
\text { s }\end{array}$ & $\begin{array}{l}\mathrm{n} \\
\mathrm{o}\end{array}$ & $\%$ & $\begin{array}{l}\mathrm{S} \\
\mathrm{o} \\
\mathrm{m} \\
\mathrm{e} \\
\mathrm{Ti} \\
\mathrm{m} \\
\mathrm{e}\end{array}$ & $\begin{array}{l}\mathrm{n} \\
\mathrm{o}\end{array}$ & $\%$ & $\begin{array}{l}\mathrm{R} \\
\mathrm{a} \\
\mathrm{r} \\
\mathrm{e}\end{array}$ & $\begin{array}{l}\mathrm{n} \\
\mathrm{o}\end{array}$ & $\%$ & $\begin{array}{l}\mathrm{n} \\
\mathrm{e} \\
\mathrm{v} \\
\mathrm{er}\end{array}$ & $\begin{array}{l}\mathrm{n} \\
\mathrm{o}\end{array}$ & $\%$ & $\begin{array}{l}\mathrm{T} \\
\mathrm{o} \\
\mathrm{t} \\
\mathrm{a} \\
\mathrm{l}\end{array}$ & $\%$ \\
\hline $\begin{array}{l}\text { Feel } \\
\text { grief }\end{array}$ & & $\begin{array}{l}1 \\
2 \\
5\end{array}$ & $\begin{array}{l}2 \\
3 \\
7\end{array}$ & & $\begin{array}{l}2 \\
1 \\
7\end{array}$ & $\begin{array}{l}4 \\
1 \\
1 \\
1\end{array}$ & & $\begin{array}{l}9 \\
6\end{array}$ & $\begin{array}{l}1 \\
8 \\
2\end{array}$ & & $\begin{array}{l}9 \\
0\end{array}$ & $\begin{array}{l}1 \\
7 \\
0 \\
0\end{array}$ & $\begin{array}{l}5 \\
2 \\
8\end{array}$ & $\begin{array}{l}1 \\
0 \\
0\end{array}$ \\
\hline $\begin{array}{l}\text { Feel } \\
\text { stigma }\end{array}$ & & 8 & $\begin{array}{l}1 \\
5\end{array}$ & & $\begin{array}{l}2 \\
3\end{array}$ & $\begin{array}{l}4 \\
4\end{array}$ & & $\begin{array}{l}6 \\
0\end{array}$ & $\begin{array}{l}1 \\
1 \\
4 \\
4\end{array}$ & & $\begin{array}{l}4 \\
3 \\
7\end{array}$ & $\begin{array}{l}8 \\
2 \\
8 \\
8\end{array}$ & $\begin{array}{l}5 \\
2 \\
8\end{array}$ & $\begin{array}{l}1 \\
0 \\
0\end{array}$ \\
\hline $\begin{array}{l}\text { Feel } \\
\text { isolated }\end{array}$ & & $\begin{array}{l}7 \\
0\end{array}$ & $\begin{array}{l}1 \\
3 \\
3 \\
3\end{array}$ & & $\begin{array}{l}2 \\
0 \\
3\end{array}$ & $\begin{array}{l}3 \\
8 \\
4 \\
4\end{array}$ & & $\begin{array}{l}1 \\
2 \\
2\end{array}$ & $\begin{array}{l}2 \\
3 \\
\\
1\end{array}$ & & $\begin{array}{l}1 \\
3 \\
3\end{array}$ & $\begin{array}{l}2 \\
5 \\
2 \\
2\end{array}$ & $\begin{array}{l}5 \\
2 \\
8\end{array}$ & $\begin{array}{l}1 \\
0 \\
0\end{array}$ \\
\hline $\begin{array}{l}\text { Feel } \\
\text { weak }\end{array}$ & & $\begin{array}{l}3 \\
3\end{array}$ & $\begin{array}{l}6 \\
3\end{array}$ & & $\begin{array}{l}1 \\
4 \\
7\end{array}$ & $\begin{array}{l}2 \\
7 \\
8 \\
8\end{array}$ & & $\begin{array}{l}1 \\
1 \\
0\end{array}$ & $\begin{array}{l}2 \\
0 \\
8 \\
8\end{array}$ & & $\begin{array}{l}2 \\
3 \\
8\end{array}$ & $\begin{array}{l}4 \\
5 \\
. \\
1\end{array}$ & $\begin{array}{l}5 \\
2 \\
8\end{array}$ & $\begin{array}{l}1 \\
0 \\
0\end{array}$ \\
\hline $\begin{array}{l}\text { Feel } \\
\text { indiffer } \\
\text { ent }\end{array}$ & & $\begin{array}{l}1 \\
4\end{array}$ & $\begin{array}{l}2 \\
7\end{array}$ & & $\begin{array}{l}1 \\
0 \\
6\end{array}$ & $\begin{array}{l}2 \\
0 \\
. \\
1\end{array}$ & & $\begin{array}{l}1 \\
1 \\
4\end{array}$ & $\begin{array}{l}2 \\
1 \\
6\end{array}$ & & $\begin{array}{l}2 \\
9 \\
4\end{array}$ & $\begin{array}{l}5 \\
5 \\
7 \\
7\end{array}$ & $\begin{array}{l}5 \\
2 \\
8\end{array}$ & $\begin{array}{l}1 \\
0 \\
0\end{array}$ \\
\hline $\begin{array}{l}\text { Feel } \\
\text { broken }\end{array}$ & & $\begin{array}{l}1 \\
7\end{array}$ & $\begin{array}{l}3 \\
2 \\
2\end{array}$ & & $\begin{array}{l}9 \\
1\end{array}$ & $\begin{array}{l}1 \\
7 \\
. \\
2\end{array}$ & & $\begin{array}{l}1 \\
0 \\
3\end{array}$ & $\begin{array}{l}1 \\
9 \\
5 \\
5\end{array}$ & & $\begin{array}{l}3 \\
1 \\
7\end{array}$ & $\begin{array}{l}6 \\
0 \\
0 \\
0\end{array}$ & $\begin{array}{l}5 \\
2 \\
8\end{array}$ & $\begin{array}{l}1 \\
0 \\
0\end{array}$ \\
\hline $\begin{array}{l}\text { Feel } \\
\text { sorrow }\end{array}$ & & $\begin{array}{l}3 \\
4\end{array}$ & $\begin{array}{l}6 \\
4\end{array}$ & & $\begin{array}{l}1 \\
3 \\
7\end{array}$ & $\begin{array}{l}2 \\
5 \\
. \\
9\end{array}$ & & $\begin{array}{l}9 \\
9\end{array}$ & $\begin{array}{l}1 \\
8 \\
8\end{array}$ & & $\begin{array}{l}2 \\
5 \\
8\end{array}$ & $\begin{array}{l}4 \\
8 \\
9 \\
9\end{array}$ & $\begin{array}{l}5 \\
2 \\
8\end{array}$ & $\begin{array}{l}1 \\
0 \\
0\end{array}$ \\
\hline $\begin{array}{l}\text { Weepin } \\
\mathrm{g} \\
\text { without } \\
\text { reason }\end{array}$ & & $\begin{array}{l}1 \\
5\end{array}$ & $\begin{array}{l}2 \\
8 \\
8\end{array}$ & & $\begin{array}{l}5 \\
4\end{array}$ & $\begin{array}{l}1 \\
0 \\
2 \\
2\end{array}$ & & $\begin{array}{l}7 \\
4\end{array}$ & $\begin{array}{l}1 \\
4 \\
. \\
0\end{array}$ & & $\begin{array}{l}3 \\
8 \\
5\end{array}$ & $\begin{array}{l}7 \\
2 \\
9 \\
9\end{array}$ & $\begin{array}{l}5 \\
2 \\
8\end{array}$ & $\begin{array}{l}1 \\
0 \\
0\end{array}$ \\
\hline $\begin{array}{l}\text { Feel } \\
\text { hesitant }\end{array}$ & & $\begin{array}{l}2 \\
5\end{array}$ & $\begin{array}{l}4 \\
7 \\
\end{array}$ & & $\begin{array}{l}1 \\
2 \\
6 \\
\end{array}$ & $\begin{array}{l}2 \\
3\end{array}$ & & $\begin{array}{l}1 \\
0 \\
7 \\
\end{array}$ & $\begin{array}{l}2 \\
0\end{array}$ & & $\begin{array}{l}2 \\
7 \\
0\end{array}$ & $\begin{array}{l}5 \\
1\end{array}$ & $\begin{array}{l}5 \\
2 \\
8 \\
\end{array}$ & $\begin{array}{l}1 \\
0 \\
0\end{array}$ \\
\hline
\end{tabular}




\begin{tabular}{|c|c|c|c|c|c|c|c|c|c|c|}
\hline & & & & 9 & & 3 & & 1 & & \\
\hline $\begin{array}{l}\text { Feel } \\
\text { hopeles } \\
\text { sness }\end{array}$ & $\begin{array}{l}2 \\
1\end{array}$ & $\begin{array}{l}4 \\
. \\
0\end{array}$ & $\begin{array}{l}8 \\
4\end{array}$ & $\begin{array}{l}1 \\
5 \\
9\end{array}$ & $\begin{array}{l}8 \\
2\end{array}$ & $\begin{array}{l}1 \\
5\end{array}$ & $\begin{array}{l}3 \\
4 \\
1\end{array}$ & $\begin{array}{l}6 \\
4\end{array}$ & $\begin{array}{l}5 \\
2 \\
8\end{array}$ & $\begin{array}{l}1 \\
0 \\
0\end{array}$ \\
\hline $\begin{array}{l}\text { Feel } \\
\text { insecur } \\
\mathrm{e}\end{array}$ & $\begin{array}{l}3 \\
4\end{array}$ & $\begin{array}{l}6 \\
4\end{array}$ & $\begin{array}{l}1 \\
2 \\
3\end{array}$ & $\begin{array}{l}2 \\
3 \\
3\end{array}$ & $\begin{array}{l}1 \\
0 \\
3\end{array}$ & $\begin{array}{l}1 \\
9\end{array}$ & $\begin{array}{l}2 \\
6 \\
8\end{array}$ & $\begin{array}{l}5 \\
0 \\
8\end{array}$ & $\begin{array}{l}5 \\
2 \\
8\end{array}$ & $\begin{array}{l}1 \\
0 \\
0\end{array}$ \\
\hline $\begin{array}{l}\text { Speciou } \\
\text { sness } \\
\text { of any } \\
\text { one } \\
\text { sneeze }\end{array}$ & $\begin{array}{l}5 \\
2 \\
5\end{array}$ & $\begin{array}{l}9 \\
9 \\
4\end{array}$ & 2 & 4 & $\begin{array}{l}0 \\
0\end{array}$ & 0 & 1 & 2 & $\begin{array}{l}5 \\
2 \\
8\end{array}$ & $\begin{array}{l}1 \\
0 \\
0\end{array}$ \\
\hline $\begin{array}{l}\text { Loss of } \\
\text { confide } \\
\text { nce }\end{array}$ & $\begin{array}{l}2 \\
7\end{array}$ & $\begin{array}{l}5 \\
5 \\
1\end{array}$ & $\begin{array}{l}9 \\
8\end{array}$ & $\begin{array}{l}1 \\
8 \\
. \\
6\end{array}$ & $\begin{array}{l}1 \\
1 \\
1\end{array}$ & $\begin{array}{l}2 \\
1\end{array}$ & $\begin{array}{l}2 \\
9 \\
2\end{array}$ & $\begin{array}{l}5 \\
5\end{array}$ & $\begin{array}{l}5 \\
2 \\
8\end{array}$ & $\begin{array}{l}1 \\
0 \\
0\end{array}$ \\
\hline $\begin{array}{l}\text { Feel } \\
\text { despera } \\
\text { te }\end{array}$ & $\begin{array}{l}1 \\
9\end{array}$ & $\begin{array}{l}3 \\
6\end{array}$ & $\begin{array}{l}8 \\
2\end{array}$ & $\begin{array}{l}1 \\
5 \\
5 \\
5\end{array}$ & $\begin{array}{l}1 \\
0 \\
6\end{array}$ & $\begin{array}{l}2 \\
0\end{array}$ & $\begin{array}{l}3 \\
2 \\
1\end{array}$ & $\begin{array}{l}6 \\
0\end{array}$ & $\begin{array}{l}5 \\
2 \\
8\end{array}$ & $\begin{array}{l}1 \\
0 \\
0\end{array}$ \\
\hline $\begin{array}{l}\text { Feel } \\
\text { obsessi } \\
\text { on }\end{array}$ & $\begin{array}{l}3 \\
7\end{array}$ & $\begin{array}{l}7 \\
. \\
0\end{array}$ & $\begin{array}{l}2 \\
0 \\
3\end{array}$ & $\begin{array}{l}3 \\
8 \\
4 \\
4\end{array}$ & $\begin{array}{l}1 \\
2 \\
2\end{array}$ & $\begin{array}{l}2 \\
3 \\
1\end{array}$ & $\begin{array}{l}2 \\
0 \\
3\end{array}$ & $\begin{array}{l}3 \\
8\end{array}$ & $\begin{array}{l}5 \\
2 \\
8\end{array}$ & $\begin{array}{l}1 \\
0 \\
0\end{array}$ \\
\hline $\begin{array}{l}\text { Feel } \\
\text { panic }\end{array}$ & $\begin{array}{l}2 \\
6\end{array}$ & $\begin{array}{l}4 \\
9\end{array}$ & $\begin{array}{l}9 \\
6\end{array}$ & $\begin{array}{l}1 \\
8 \\
5 \\
2\end{array}$ & $\begin{array}{l}1 \\
1 \\
0\end{array}$ & $\begin{array}{l}2 \\
0\end{array}$ & $\begin{array}{l}2 \\
9 \\
6\end{array}$ & $\begin{array}{l}5 \\
6\end{array}$ & $\begin{array}{l}5 \\
2 \\
8\end{array}$ & $\begin{array}{l}1 \\
0 \\
0\end{array}$ \\
\hline $\begin{array}{l}\text { Suffer } \\
\text { sleep } \\
\text { disturb } \\
\text { ances }\end{array}$ & & & & & & & & & & \\
\hline
\end{tabular}

Table 12 above showed the psychosocial reactions of respondents to covid19, the majority feel suspicious when someone sneeze, feel isolated, feel grief and feel obsessed.

\section{Discussion}

The objective of the study is for discover the psychosocial reactions to corona virus 2019(covid19) among the individual in the Arabic world. The respondents of this study are 528 persons, 398 are females and 130 are males. Half of the respondents age from the age group $41-60$ years old, which constitute $47.1 \%$. The majority of them from Urban origin $97.9 \%$, their occupation vary $23.9 \%$ are housewives and this 
is expected because the female constitute the great number of the study. The highest respondents from Sudan and Saudi Arabia, which constitute $72.7 \%$. When we look to the psychosocial reactions to covid19, the significant among the age group 51-60 years old. This is logical; that age group are the more considered as risk group to covid19 infection. The place of origin influence the individual behaver, the early year of the person life has great influences on the person future according to many psychological theories (psychodynamic as an example). In this study the place of origin play significance difference in the psychosocial reactions to covid19 in favor to urban origin, this obvious, the majority of the respondents from urban origin. Those who work in private sector, they more concern with and sensitive to covid19 issues simply because their economic conditions is more better than the other sectors, they can afford sensitizers, masks, good quality of food, can follow the information pertaining to covid19 thus their psychosocial reaction is high. Although, Sudan and Saudi Arabia among the highest respondents still there is no significance differences in psychosocial reactions to covid19 it may attributed to the similarities between the two communities. The more respondents from these two countries because the researchers origin from them, and they distributed to the groups they know via the internet. Almost great number of the respondents, $97.5 \%$ have no the disease nor live with covid19 patients or interact with them. This, the psychosocial reactions is more exaggerated $23.7 \%$ and $41.1 \%$ always and sometime feel grief respectively.Covid 19 associated with stigma for the patients and their families and relatives, this clear in this study $82.8 \%$ never feel stigmatized because they are not have the disease to themselves or the their relatives. Still feeling of isolation is there; half of the respondents feel isolated $20.3 \%$ and $29.5 \%$ of the respondents feel isolated always and sometime respectively when hear about covid19. Usually when an individual received negative impact and evaluation from the society, he or she have several negative feeling like hate, but still because the majority of the respondents have no covid19 experiences, hate never find their way to them $73.9 \%$ never feel hate.

Anxiety is the natural feeling and psychological reaction to the unknown problems, covid19 is one of the anxiety source trigger to people. Countries, governments and different agencies, so $13.3 \%$ and $38.4 \%$ feel anxious always and sometime respectively. The effect of covid19 all over the world cause weakness in different life aspect with no exception. Nevertheless, in this study because the majority have no covd19 experiences half of them never feel weak, and indifference occurred for those who feel desperate weak, hate, but in this study $55.7 \%$ never feel indifference simply because as mentioned several times they did not have covid19 experiences. Forty eight point nine percent feel broken, $51.5 \%$ never feel hesitant, and $50.8 \%$ never feel insecure, $55.3 \%$ lack of confidence. However, $99.4 \%$ always feel suspicious about covid 19 and $31.4 \%$ some time feel obsess and $53.8 \%$ never suffer sleep disturbances. This result cannot be generalized to completely Arabic world population but it can be an indicator to how far individual behavior will change due to covid19, further behavioral studies can in deepen these issues. In addition this study took please on 
march at the beginning of this outbreak, if it replicated it can show different results.

\section{Conclusion and Recommendations}

This study aimed at investigating the psychosocial reaction to covid19 among a sample of 598 individual from the Arabic world, they filling in an open-ended questions, asking mainly about the psychological and social reactions when hearing about covid19. The result showed that there is no sever psychological reaction to covd19 except in feeling stigma, isolation, anxious and obsessions, these logical for people who have no direct experiences of covid19. The majority of the respondents have no experiences with the pandemic. These mentioned reactions mainly expected among any individual due to the information delivered to people about covid19. The result will not generalized but it has an important indicator of psychosocial reaction especially this study conducted earlier at the start of the pandemic. Further studies can concentrate on the infected psychosocial reactions, those who live with infected psychosocial reaction.

\section{References}

[1] a practical guide.

[2] Bai, Y., Lin, C. C., Lin, C. Y., Chen, J. Y., Chue, C. M., \& Chou, P. (2004). Survey of stress reactions among health care workers involved with the SARS outbreak. Psychiatric Services, 55(9), 1055-1057.

[3] Barbisch, D., Koenig, K. L., \& Shih, F. Y. (2015). Is there a case for quarantine? Perspectives from SARS to Ebola. Disaster medicine and public health preparedness, 9(5), 547-553.

[4] Blendon, R. J., Benson, J. M., DesRoches, C. M., Raleigh, E., \& Taylor-Clark, K. (2004). The public's response to severe acute respiratory syndrome in Toronto and the United States. Clinical infectious diseases, 38(7), 925-931.

[5] Braunack-Mayer, A., Tooher, R., Collins, J. E., Street, J. M., \& Marshall, H. (2013). Understanding the school community's response to school closures during the H1N1 2009 influenza pandemic. BMC public health, 13(1), 1-15.

[6] Caleo, G., Duncombe, J., Jephcott, F., Lokuge, K., Mills, C., Looijen, E., ... \& Greig, J. (2018). The factors affecting household transmission dynamics and community compliance with Ebola control measures: a mixed-methods study in a rural village in Sierra Leone. BMC public health, 18(1), 1-13.

[7] Cava, M. A., Fay, K. E., Beanlands, H. J., McCay, E. A., \& Wignall, R. (2005). The experience of quarantine for individuals affected by SARS in Toronto. Public Health Nursing, 22(5), 398-406.

[8] Desclaux, A., Badji, D., Ndione, A. G., \& Sow, K. (2017). Accepted monitoring or endured quarantine? Ebola contacts' perceptions in Senegal. Social science \& medicine, 178, 38-45.

[9] DiGiovanni, C., Conley, J., Chiu, D., \& Zaborski, J. (2004). Factors influencing compliance with quarantine in Toronto during the 2003 SARS outbreak. Biosecurity and bioterrorism: biodefense strategy, practice, and 
science, 2(4), 265-272.

[10] Hawryluck, L., Gold, W. L., Robinson, S., Pogorski, S., Galea, S., \& Styra, R. (2004). SARS control and psychological effects of quarantine, Toronto, Canada. Emerging infectious diseases, 10(7), 1206.

[11] Hong, X., Currier, G. W., Zhao, X., Jiang, Y., Zhou, W., \& Wei, J. (2009). Posttraumatic stress disorder in convalescent severe acute respiratory syndrome patients: a 4-year follow-up study.General hospital psychiatry, 31(6), 546-554.

[12] Jeong, H., Yim, H. W., Song, Y. J., Ki, M., Min, J. A., Cho, J., \& Chae, J. H. (2016). Mental health status of people isolated due to Middle East Respiratory Syndrome. Epidemiology and health, 38.

[13] Lee, S., Chan, L. Y., Chau, A. M., Kwok, K. P., \& Kleinman, A. (2005). The experience of SARS-related stigma at Amoy Gardens. Social science \& medicine, 61(9), 2038-2046.

[14] Liu, X., Kakade, M., Fuller, C. J., Fan, B., Fang, Y., Kong, J., ... \& Wu, P. (2012). Depression after exposure to stressful events: lessons learned from the severe acute respiratory syndrome epidemic. Comprehensive psychiatry, 53(1), 1523.

[15] Manuell, M. E., \& Cukor, J. (2011). Mother Nature versus human nature: public compliance with evacuation and quarantine. Disasters, 35(2), 417-442.

[16] Marjanovic, Z., Greenglass, E. R., \& Coffey, S. (2007). The relevance of psychosocial variables and working conditions in predicting nurses' coping strategies during the SARS crisis: an online questionnaire survey. International journal of nursing studies, 44(6), 991-998.

[17] Maunder, R., Hunter, J., Vincent, L., Bennett, J., Peladeau, N., Leszcz, M., ... \& Mazzulli, T. (2003). The immediate psychological and occupational impact of the 2003 SARS outbreak in a teaching hospital. Cmaj, 168(10), 1245-1251.

[18] Mihashi, M., Otsubo, Y., Yinjuan, X., Nagatomi, K., Hoshiko, M., \& Ishitake, T. (2009). Predictive factors of psychological disorder development during recovery following SARS outbreak. Health Psychology, 28(1), 91.

[19] Miles, S. H. (2015). Kaci Hickox: public health and the politics of fear. The American Journal of Bioethics, 15(4), 17-19. http://www.bioethics.net/2014/11/kacihickoxpublichealthandthe politicsoffear/

[20] Newman, K. L. (2012). Shutt up: bubonic plague and quarantine in early modern England. Journal of social history, 45(3), 809-834.

[21] Nilima, N., Kaushik, S., Tiwary, B., \& Pandey, P. K. (2021). Psycho-social factors associated with the nationwide lockdown in India during COVID-19 pandemic. Clinical Epidemiology and Global Health, 9, 47-52.

[22] Nilima, N., Kaushik, S., Tiwary, B., \& Pandey, P. K. Clinical Epidemiology and Global Health.

[23] Public Health England. Novel coronavirus (2019nCoV) - what you need to 
know. 2020. https://publichealthmatters.blog.gov.uk/ 2020/01/23/wuhannovelcoronaviruswhatyouneedtoknow/.

[24] Rubin, G. J., \& Wessely, S. (2020). The psychological effects of quarantining a city. $B m j, 368$.

[25] Tricco, A. C., Langlois, E., Straus, S. E., \& World Health Organization. (2017). Rapid reviews to strengthen health policy and systems: a practical guide. World Health Organization.

\section{Appendix}

Table Questions fill in time duration

\begin{tabular}{|c|c|c|c|c|c|}
\hline & & Frequency & Percent & $\begin{array}{l}\text { Valid } \\
\text { Percent }\end{array}$ & $\begin{array}{l}\text { Cumulative } \\
\text { Percent }\end{array}$ \\
\hline \multirow[t]{13}{*}{ Valid } & $\begin{array}{l}\text { 22-Apr- } \\
2020\end{array}$ & 191 & 36.2 & 36.2 & 36.2 \\
\hline & $\begin{array}{l}\text { 23-Apr- } \\
2020\end{array}$ & 93 & 17.6 & 17.6 & 53.8 \\
\hline & $\begin{array}{l}\text { 24-Apr- } \\
2020\end{array}$ & 17 & 3.2 & 3.2 & 57.0 \\
\hline & $\begin{array}{l}\text { 25-Apr- } \\
2020\end{array}$ & 35 & 6.6 & 6.6 & 63.6 \\
\hline & $\begin{array}{l}\text { 26-Apr- } \\
2020\end{array}$ & 56 & 10.6 & 10.6 & 74.2 \\
\hline & $\begin{array}{l}\text { 27-Apr- } \\
2020\end{array}$ & 37 & 7.0 & 7.0 & 81.3 \\
\hline & $\begin{array}{l}\text { 28-Apr- } \\
2020\end{array}$ & 17 & 3.2 & 3.2 & 84.5 \\
\hline & $\begin{array}{l}\text { 29-Apr- } \\
2020\end{array}$ & 36 & 6.8 & 6.8 & 91.3 \\
\hline & $\begin{array}{l}\text { 30-Apr- } \\
2020\end{array}$ & 2 & .4 & .4 & 91.7 \\
\hline & $\begin{array}{l}\text { 01-May- } \\
2020\end{array}$ & 1 & .2 & .2 & 91.9 \\
\hline & $\begin{array}{l}\text { 02-May- } \\
2020\end{array}$ & 1 & .2 & .2 & 92.0 \\
\hline & $\begin{array}{l}\text { 08-May- } \\
2020\end{array}$ & 1 & .2 & .2 & 92.2 \\
\hline & $\begin{array}{l}\text { 09-May- } \\
2020\end{array}$ & 1 & .2 & .2 & 92.4 \\
\hline
\end{tabular}




\begin{tabular}{|l|l|l|l|l|l|}
\hline & $\begin{array}{l}10-\text { May- } \\
2020\end{array}$ & 2 & .4 & .4 & 92.8 \\
\hline & $\begin{array}{l}\text { 11-May- } \\
2020\end{array}$ & 23 & 4.4 & 4.4 & 97.2 \\
\hline $\begin{array}{l}12-\text { May- } \\
2020\end{array}$ & 14 & 2.7 & 2.7 & 99.8 \\
\hline $\begin{array}{l}13-\text { May- } \\
2020\end{array}$ & 1 & .2 & .2 & 100.0 \\
\hline & Total & 528 & 100.0 & 100.0 & \\
\hline
\end{tabular}

Notice: the most frequent responses in 22 April 2020 which equal 191 and 36.2\% . 